\title{
Hydro Magnetic Effect on Thread Stretching Surface with Mounted Obstacles in Porous Medium
}

\author{
MOHAMMAD MOHSEN PEIRAVI*, POOYA PASHA, DAVOOD DOMAIRRY GANJI \\ Department of mechanical Engineering \\ Babol Noshirvani University of Technology \\ P.O. Box47166-85635, Babol \\ IRAN
}

\begin{abstract}
In this paper, Finite element Model is applied for investigation of fluid flow over a stretching sheet in existence of magnetic field. Finite element method is applied to find the influence of melting heat transfer on fluid flow behavior over a stretching sheet in presence of magnetic field. we investigated the flow of fluid flowing through the fins plate under the influence of the magnet. The fins were on the board and the end of the plate. In the case of chamfer fins, the maximum temperature variation is observed. In this fins, the maximum temperature of $T=2.5$ and minimum temperature is $T=3$. in general, we conclude that the temperature flow around the rectangular fins has a maximum value than 2 other modes. In triangular fins, the fluid temperature vector around the fins has more intensity than other modes and the temperature gradient around it is larger than the previous one and the fluid flow at the end of the plate also has more temperature than the Rectangular fins. The maximum amount of fluid concentration has been observed around the first fin of chamfer mode in range of $\mathrm{X}=0.05$ to $\mathrm{X}=0.1$. In general, the fluid concentration around the triangular fins is higher than other modes. the maximum amount of fluid concentration is found in the triangular fins on the surface. Their concentration from the first fin reaches a value of 2.5 and in the last fin at a value of 1.4.
\end{abstract}

Key-Words: - Finite element method; Hydro magnetic effect; Mounted obstacles; Porous medium; Stretching sheet; Thread surface.

Received: January 5, 2021. Revised: June 12, 2021. Accepted: July 1, 2021. Published: July 12, 2021.

\section{Introduction}

In this project, Finite element Model is applied for investigation of fluid flow over a stretching sheet in existence of magnetic field. Finite element method is applied to find the influence of melting heat transfer on fluid flow behavior over a stretching sheet in presence of magnetic field.

Additionally, the melting point is often used to predict the partition behavior of a chemical between the solid and gaseous phases. No melting point test is necessary for each chemical. Ganji et al. [1] investigated of threads surface integrity manufactured by cutting insert and with internal thread rolling head. The surface micro-morphology findings can be used in research to improve the lifetime of similar rolled threads and mathematical modeling of tool-workpiece lubrication processes. Maddah et al. [2] investigated Fractal geometry of internal thread surfaces manufactured by cutting tap and rolling tap. The application of fractal geometry in internal threads surface characterization to disclose fault fine structures of the internal thread geometry caused by the manufacturing process is briefly presented in this work. Adamo et al. [3] studied Fast and straightforward in-situ synthesis of gold nanoparticles on a thread-based microfluidic device for application in surface-enhanced Raman scattering detection. For the first time, cotton threads formed a microfluidic system that was used to conduct a fast and simple Turkevich synthesis of gold nanoparticles (AuNPs) using minute amounts of chemicals. Yen et al [4] studied and examined the topic that Cone beam computed tomography accuracy in determining the thickness of hardtissue-mimicking material close to various implant thread surfaces. Using an in vitro model, assess the measuring accuracy of hard-tissue thicknesses close to dental implants using various thread patterns on images generated from cone beam computed tomography (CBCT). Abbasi et al. [5] investigated the effects of hybrid nanoparticles on solid-liquid phase change process in a Y-shaped fin-assisted LHTESS by means of FEM. The unstable heat and mass transfer during the solidification process is simulated using the Finite Element Method (FEM). The results showed that increasing the fin length and fin bifurcation angle reduces the average temperature and total energy of the system while increasing the solid percent. Seyyedi et al. [6] researched entropy generation in a square inclined cavity using control volume finite element method with aided quadratic Lagrange interpolation 
functions. The results reveal that when the Hartmann number increases, the entropy generation number increases as well, with a maximum value for a given inclination angle. In addition, at low values of Rayleigh number, ECOP rises as the Hartmann number rises. Peiravi et al. [7] researched electromagnetic and electrical modeling by the finite element method. The finite element approach is based on energy minimization in the solution of physical problems; in this case, electromagnetic energy is minimized. Paul et al. [8] investigated Finite Element Method of Analyzing Steady Seepage with a Free Surface. The approach can be used on porous heterogeneous media with complicated geometric boundaries and arbitrary degrees of anisotropy. It can handle situations with a discontinuous free surface and portions of the free surface that are vertical or nearly vertical. Sindhu et al. [9] researched Simulation of $\mathrm{Cu}$ : Y-Alooh/water in a microchannel heat sink by dint of porous media approach. Alinejad et al. [10] investigated Heat generation /absorption on MHD stagnation flow of nanofluid towards a porous stretching sheet with prescribed surface heat flux. We describe an inquiry into MHD stagnation-point flow of a nanofluid via a heated porous stretched sheet with suction or blowing circumstances and a predefined surface heat flux in this study. The transport equations include Brownian motion and thermophoresis effects. Peiravi et al. [11] researched Hydrothermal analysis of MHD squeezing mixture fluid suspended by hybrid nanoparticles between two parallel plates. The result demonstrates that the Akbari-Ganji approach and the numerical method accord well. For such issues, Akbari- Ganji's method can be effective, and we can observe that the difference in velocity and thermal profiles between AGM and the numerical method is less than $1 \%$. An increase in the squeeze number and Hartman number lowered the velocity profile. Ghadikolaei et al. [12] researched MHD boundary layer analysis for micropolar dusty fluid containing Hybrid nanoparticles $\left(\mathrm{Cu}^{-\mathrm{A}} \mathrm{2} \mathrm{O} 3\right)$ over a porous medium. The flow and heat transfer of non-Newtonian micropolar dusty fluid suspended $\mathrm{Cu}-\mathrm{Al} 2 \mathrm{O} 3$ Hybrid nanoparticles past a stretching sheet in the presence of non-linear thermal radiation, variable thermal conductivity, and various nanoparticle shapes (Bricks, Cylinders, Platelets, and Blades) are studied in this paper.

The application of a Finite Element Model to the research of fluid flow over a stretching sheet in the presence of a magnetic field is the focus of this project. The impact of melting heat transfer on fluid flow behavior over a stretching sheet in the presence of a magnetic field is investigated using the finite element approach. The method of solving the equations of linear differential equations in this paper is with the help of the Flex software. All of the parameters calculated in the FlexPDE software are finite element method and moreover, some of the essential parameters of these equations are plotted.

\section{Geometry of the Problem}

The flow of a steady magneto hydrodynamic fluid over a stretching sheet is investigated. The problem's geometry is depicted in Figures 1 and 2 and 3. Fig. 1 has 5 fins on the plane surface, with 3 blade numbers on the screen, and another 2 is placed at the bottom of the screen. Figures 2 and 3 have 3 and 4 fins on the board and are under the boundary conditions applied in Fig. 1. The stretched plate's velocity is $\left(u_{w}=a x, a>0\right)$. The ambient temperature is $T_{\text {inf }}$, and the melting temperature is $T_{m}\left(T_{\text {inf }}-b x^{2}\right)$. A vertically applied constant magnetic field is used. Because the magnetic Reynolds number is tiny, the induced magnetic field is tiny in proportion to the applied magnetic field. In the energy equation, the effects of Joule heating, viscous dissipation, and thermal radiation have been examined.

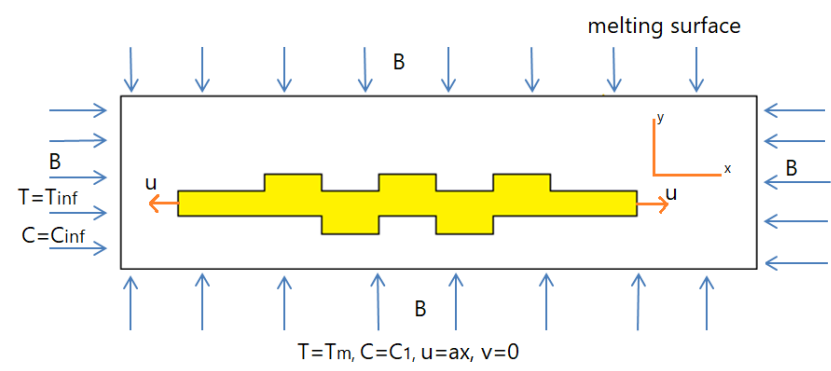

a) Stretching sheet with rectangular fins

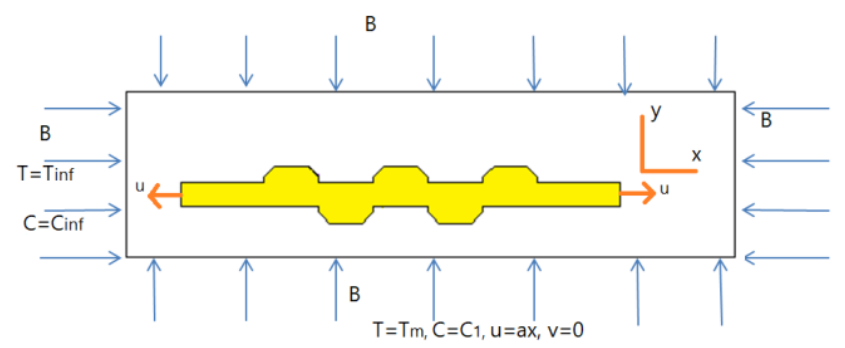

b) Stretching sheet with chamfer fins

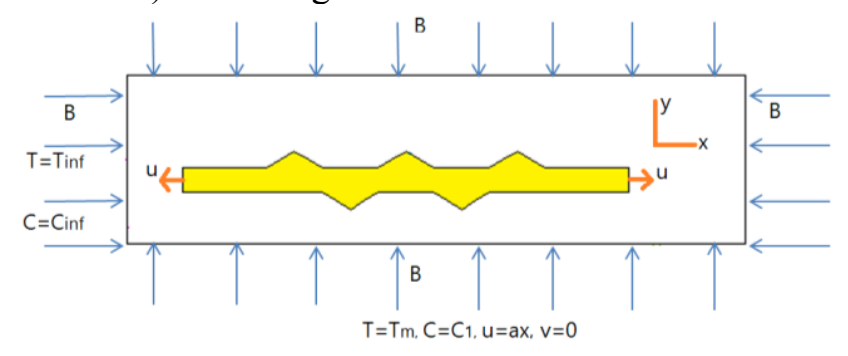


c) Stretching sheet with triangular fins

Fig. 1: schematic of hydro magnetic effect on different thread stretching sheet

The partial differential equations that govern the situation are as follows: The governing partial differential equations are:

$$
\begin{gathered}
\frac{\partial u}{\partial x}+\frac{\partial v}{\partial y}=0 \\
\rho_{f}\left(u \frac{d u}{d x}+v \frac{d u}{d y}\right)=-\frac{d p}{d x}+\mu\left(\frac{d^{2} u}{d y^{2}}\right)-\sigma B_{0}^{2} u- \\
\left(\rho C_{P}\right)_{f}\left(u \frac{d T}{d x}+v \frac{d T}{d y}\right)=k_{f}\left(\frac{d^{2} T}{d y^{2}}\right)+\sigma B_{0}^{2} u^{2}- \\
\frac{d q_{r}}{d y}+\mu\left(\frac{d u}{d y}\right)^{2}+\left(\rho C_{p}\right)_{p}\left[D_{B}\left\{\frac{d C}{d y} \cdot \frac{d T}{d y}\right\}+\right. \\
\left.\left(\frac{D_{T}}{T_{\infty}}\right)\left\{\left(\frac{d T}{d y}\right)^{2}\right\}\right] \\
u \frac{d C}{d x}+v \frac{d C}{d y}=D_{B} \frac{d^{2} C}{d y^{2}}+\left(\frac{D_{T}}{T_{\infty}}\right)\left\{\frac{d^{2} C}{d y^{2}}\right\}
\end{gathered}
$$

where the radiation heat flux $\mathrm{q}_{\mathrm{r}}$ is calculated using the Rosseland approximation, and $\mathrm{q}_{\mathrm{r}}=-\frac{4 \sigma_{e}}{3 \beta_{R}} \frac{d T^{4}}{d y}$ where $\sigma_{e}, \beta_{R}$ are the Stefan-Boltzman constant and the mean absorption coefficient. The temperature variations between the fluid phases in the flow are considered to be modest enough for $\mathrm{T}_{4}$ to be stated as a linear function of temperature. This is completed. $\mathrm{T}_{4}$ is expanded in a Taylor series around temperature Tc. Boundary conditions are:

$\left\{u=u_{w}=a x, v=0, T=T_{m}, C=\right.$

$C_{m}$, at $\left.y=0\right\}$

$\left\{T \leftrightarrow T_{\infty}, u \leftrightarrow 0, C \leftrightarrow C_{\infty}\right.$, at $\left.y \leftrightarrow \infty\right\}$

$k\left(\frac{d T}{d y}\right) \mid y=0=\rho_{f}\left(L+C_{s}\left(T_{m}-T_{0}\right)\right) v(x, 0)$

Where $\mathrm{v}$ and $\mathrm{u}$ are the velocity parameter along the $\mathrm{X}$ and $\mathrm{y}$ directions, The non-uniform latent variable $\mathrm{L}\left(=\mathrm{L}_{0} \mathrm{x}^{2}\right)$. The heat capacity of the solid surface is $\mathrm{C}_{\mathrm{s}}$, while the heat capacity of the fluid is $\mathrm{h}$. The heat transferred to the melting surface, according to the boundary condition, is equal to the heat of melting plus the sensible heat required to raise the solid temperature $\mathrm{T}\left(=\mathrm{T}_{0}-\mathrm{cx}_{2}\right)$ to its melting temperature $\mathrm{T}_{\mathrm{m}}$.

\section{Finite Element Method}

FlexPDE is a builder of scripted finite elements and numeric solver. In other words, from a script written by the user. FlexPDE carries out the necessary operation to transform a description of a partial differential equation system in a finite element model, Resolve the system and graphical and tabular results. Output of the results, FlexPDE is also a 'problem-solving environment' that executes the full range of functions required to resolve partial differential equation systems. This application is responsible for it concerns a system of tail equations in a model of finite elements and finally solve the system. One of the capabilities of the Flex software is the mesh of objects. For example, a portrait is given below:

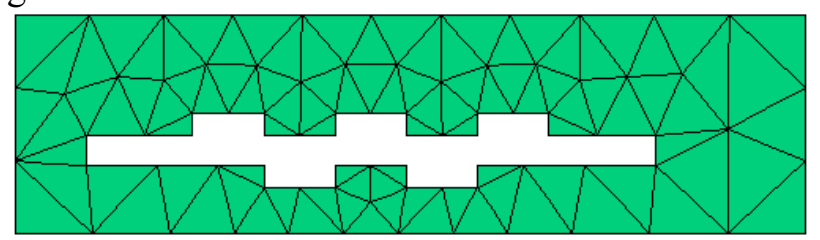

a) Stretching sheet with rectangular fins

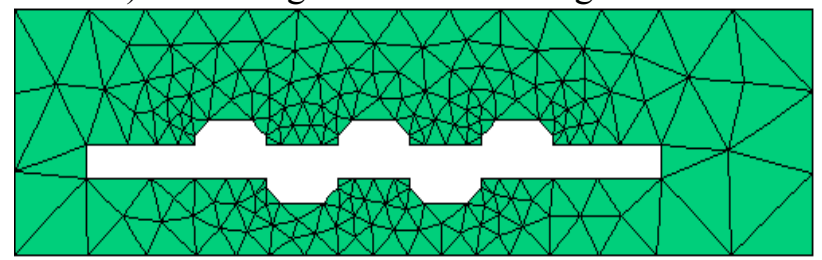

b) Stretching sheet with chamfer fins

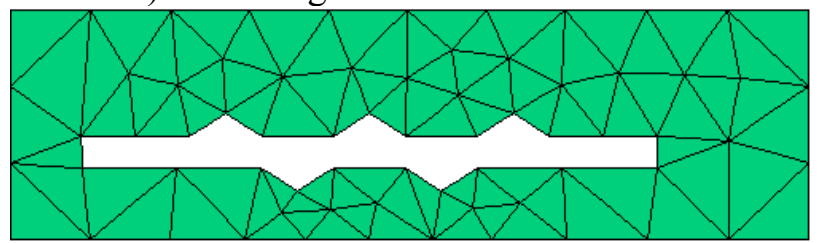

c) Stretching sheet with triangular fins

Fig. 2: schematic of mesh qualify at different thread stretching sheet

The grids of the mesh show in the chapters. The fin of chamfer mode has a better grid mesh all over the surface and the quality of mesh is better than the triangular and rectangular fins.

\section{Result and Discussion}

The present paper described a complete description of the last events in this paper. In this paper, the flow of fluid is water. The temperature of water is more than a melting surface. The temperature of water is $170 \mathrm{C}$ and temperature of melting surface is $110 \mathrm{C}$. The surface is pulled from two sides at a speed of $2 \mathrm{~m} / \mathrm{s}$. The surface density of the plane is 8400 and the specific heat value of the surface is 625. We want to show the impact of the four important and viscous parameters such as $\mathrm{T}$ and $\mathrm{C}$, and $\mathrm{v}$ and $\mathrm{u}$. The following images display the contours of velocity in the direction $\mathrm{x}$ in all different configurations of the fins:

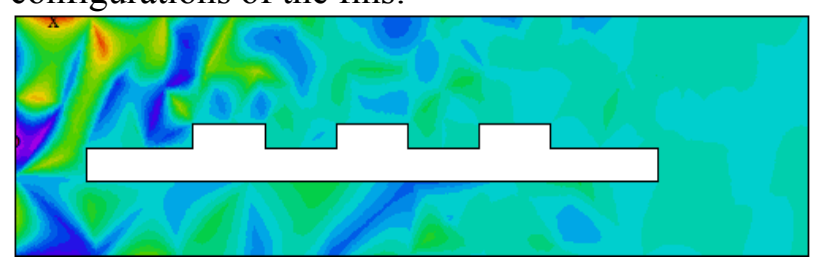




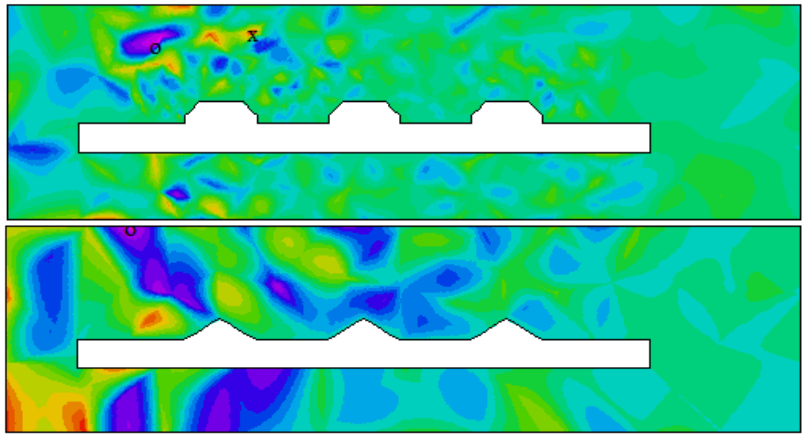

a) Stretching sheet with three fins

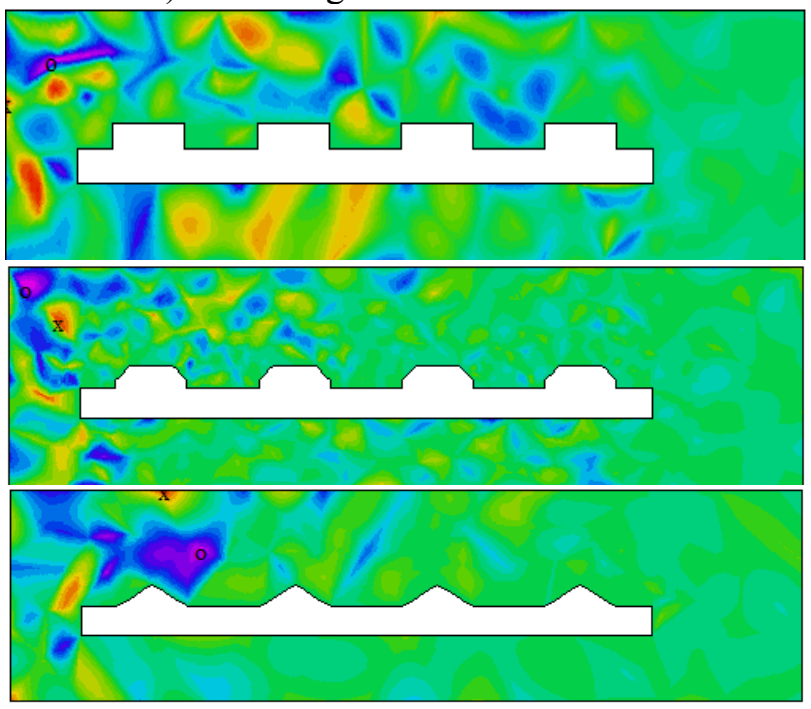

b) Stretching sheet with four fins

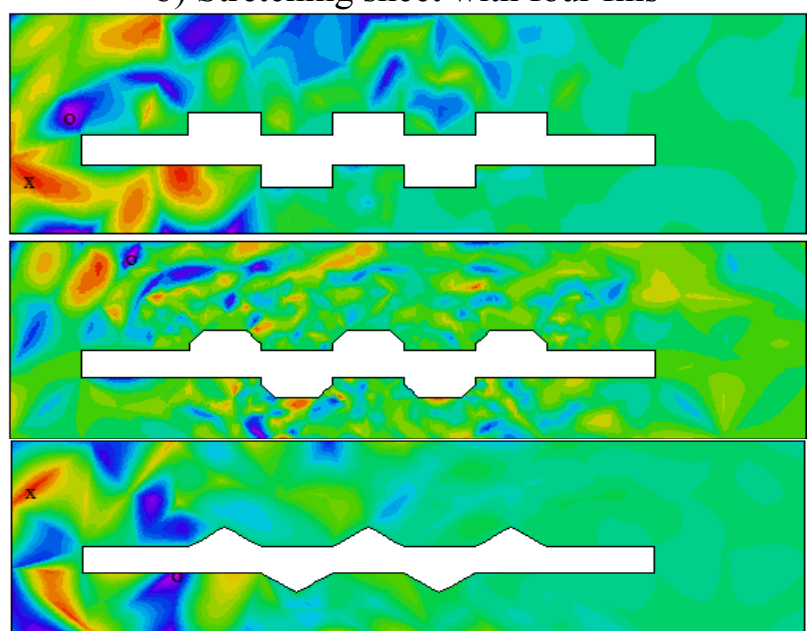

c) Stretching sheet with five fins

Fig. 3: comparison of different values of $u$ for rectangular and triangular and chamfer fins

At the top of the table, from left to right, the fin surfaces of the shape, triangular and chamfer, are produced in different states, respectively. In the first row of the table from the left to the right, there is a lot of speed at the beginning of the page, and then the fluid flow over the fins makes it very low. In comparison between 5 fin and 3 fins, the fluid flow at 5 fin is higher, and the red area in front of the plate is much higher than the 3 blade mode. In the second fin on the plane surface, the velocity fluid velocity is low, increasing the rate of speed by increasing the distance from the plane along the $y$ direction. In the second row, compared with triangular blade with 3 and 4 triangular blades, the fluid velocity around the triangular blades is seen to be much lower than the other.
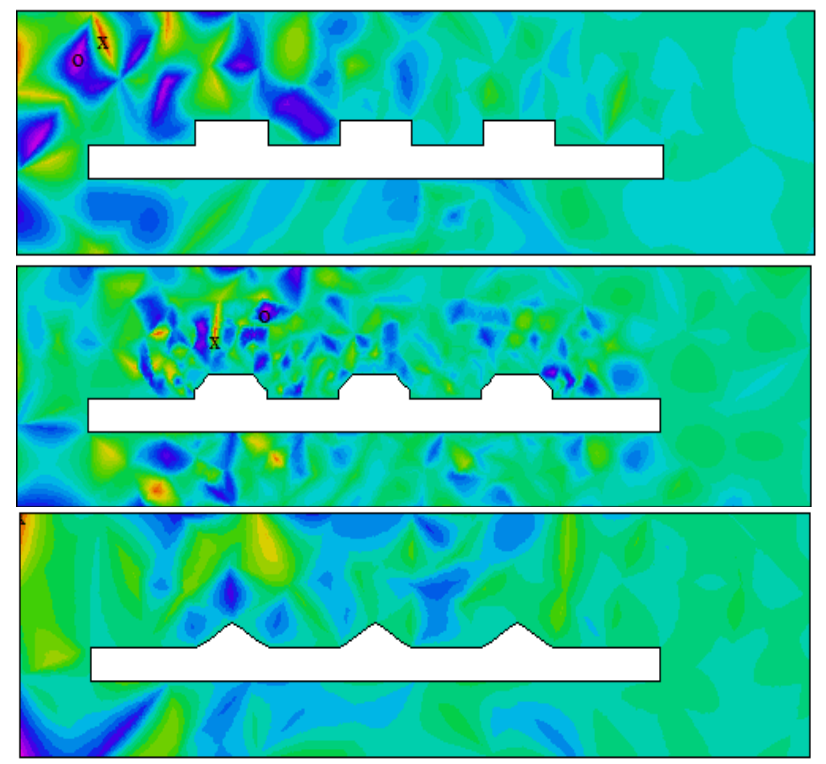

a) Stretching sheet with three fins

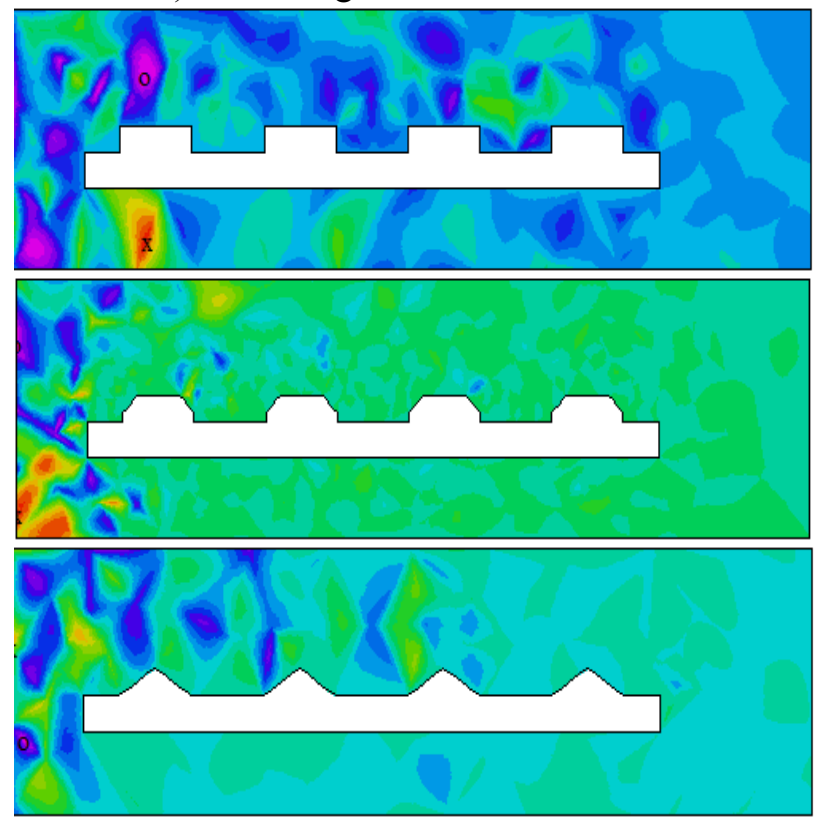

b) Stretching sheet with four fins

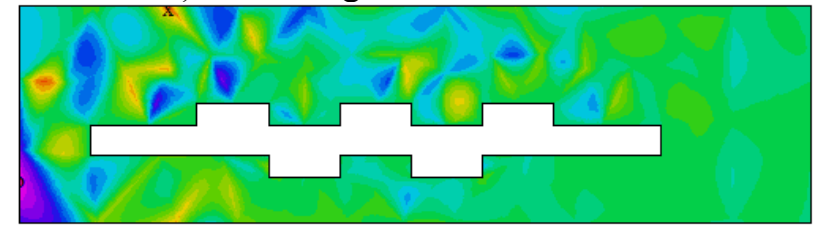




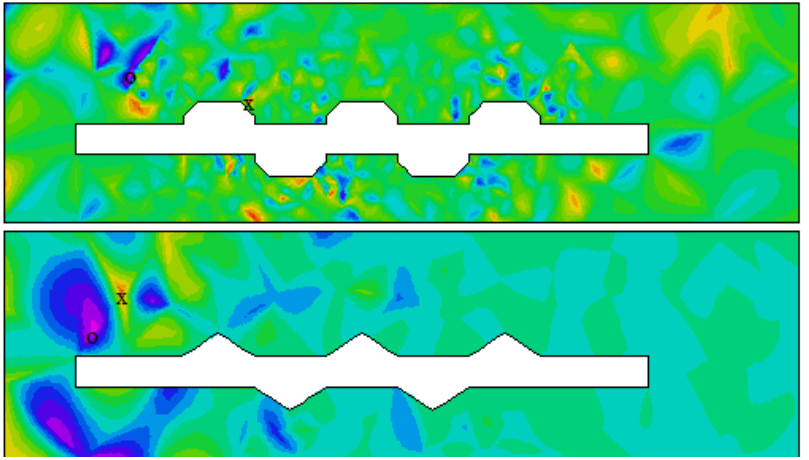

c) Stretching sheet with five fins

Fig. 4: comparison of different values of $v$ for rectangular and triangular and chamfer fins

The above images denote the velocity value of fluid (v) around the plane. First, we compare the first column of the table from the left. in the first column, the rate of fluid velocity at the first column, the rate of fluid velocity in the circle is more in the circle around the fins than 2 other modes. For example, we consider the second row of the table. in the case of 5 fins at the top and bottom of the plate, the majority of the space around the velocity of the fluid is balanced and the flow around the blades has lower rates of speed and the fluid particles have less speed than the previous modes, so that around the blades the speed of velocity changes to -20 to -40 . The fluid velocity is generally lower around the edges of the plane, and below the screen, the fluid velocity has a lower value than the bottom of the plane.

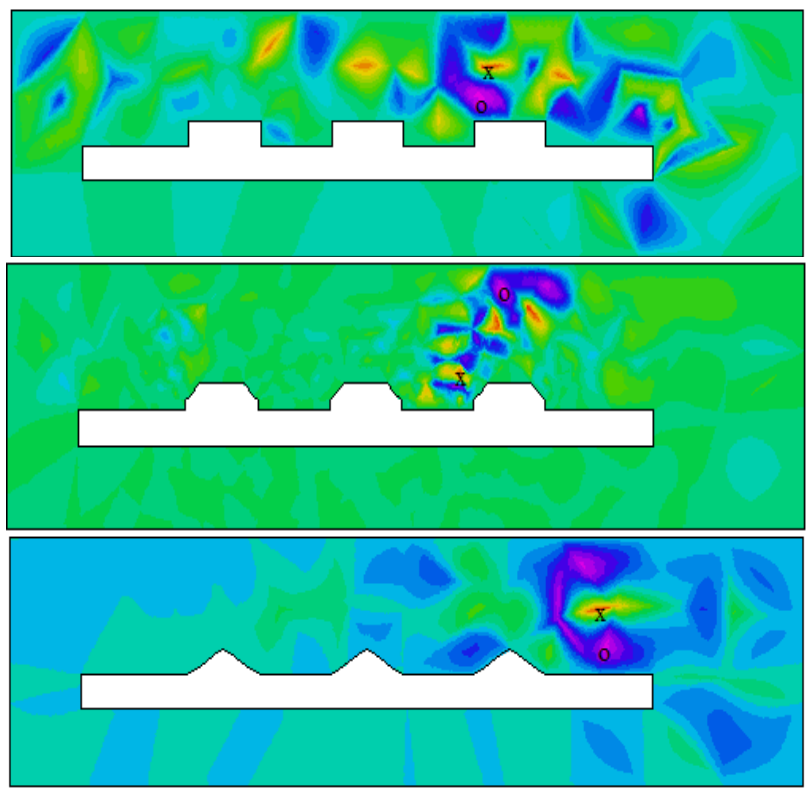

a) Stretching sheet with three fins

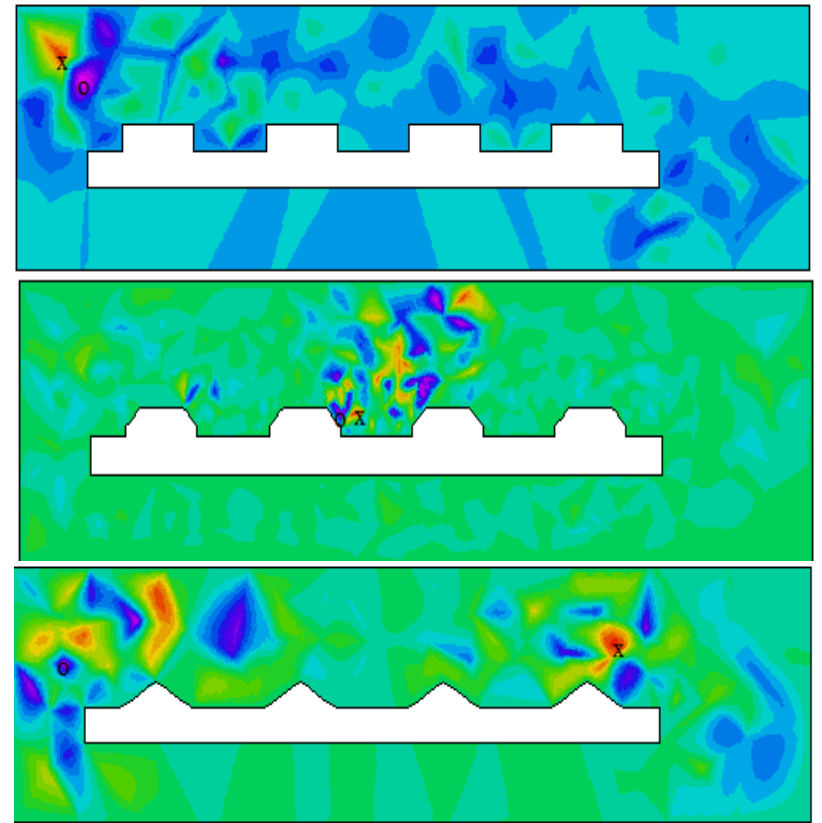

b) Stretching sheet with four fins

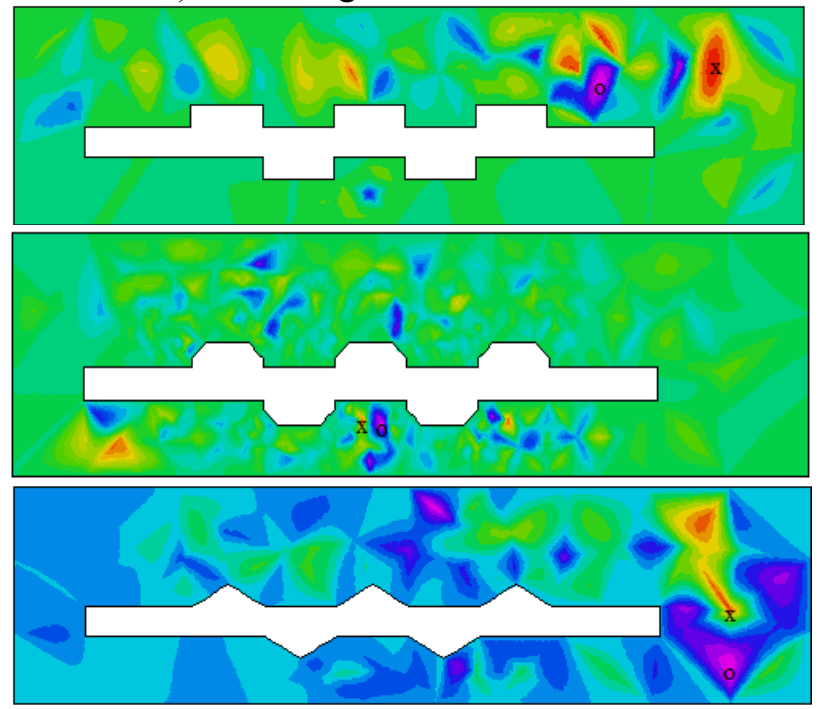

c) Stretching sheet with five fins

Fig. 5: comparison of different values of Temperature for rectangular and triangular and chamfer fins

Above, the temperature contours of the fluid around the plates with different blades show. first, we look at the fins in shape on the screen. The temperature is low around the first fin from left to right, the higher the temperature, the higher the temperature, and the same results are available in the second and third fins sticking to the board. At the end of the plane, the temperature is high. in the case of 3 rectangular fins, the temperature is decreased and the temperature gradient is low. in the case of four fins with respect to the preceding ones, the temperature around the fins is much less than the preceding ones, but in the case of 5 fins, the temperature of the fluid 
flow is balanced. We now look at the interpretation of triangular blades. the temperature of the fluid flow is very low and the fluid flow temperature is very low, but by reducing the number of blade to 3 , the fluid flow temperature is balanced and the whole set is increased. in case of 4 triangular blade, there is an area at the beginning of the board at the top of the blade where the temperature is maximum. by comparing the case of 4 rectangular fins with triangular shape, we conclude that in the case of 4 blades, the flow temperature of the fluid around the plate is much higher than the liquid temperature in the 4 rectangular blade mode. now we interpret the blades of the chamfer. In all cases, the fluid temperature is balanced and we observe less temperature drops. so that in the case of 3 blade, the fluid temperature in the second blade in the second blade is much higher than the fluid temperature in the second blade in case of 4 fins.

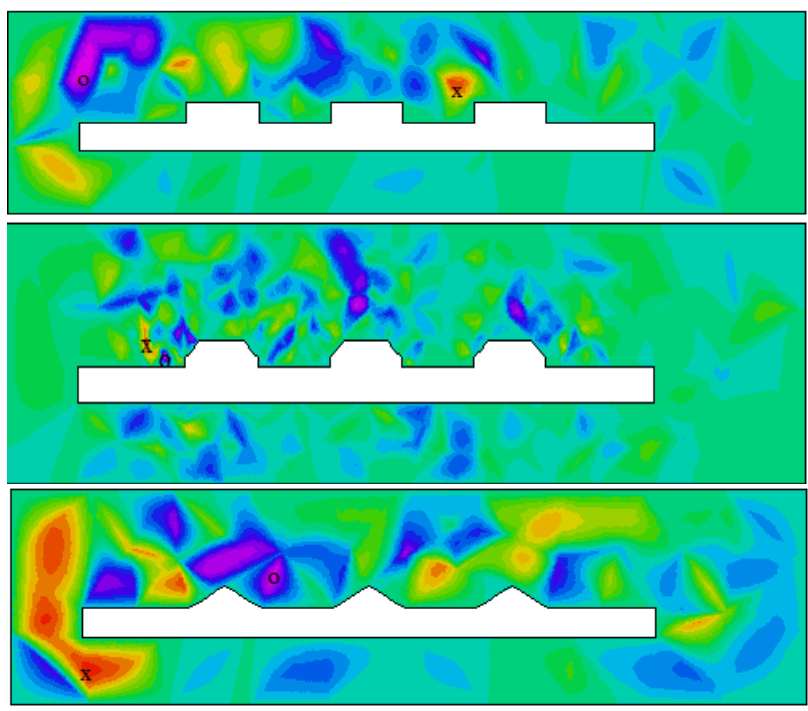

a) Stretching sheet with three fins

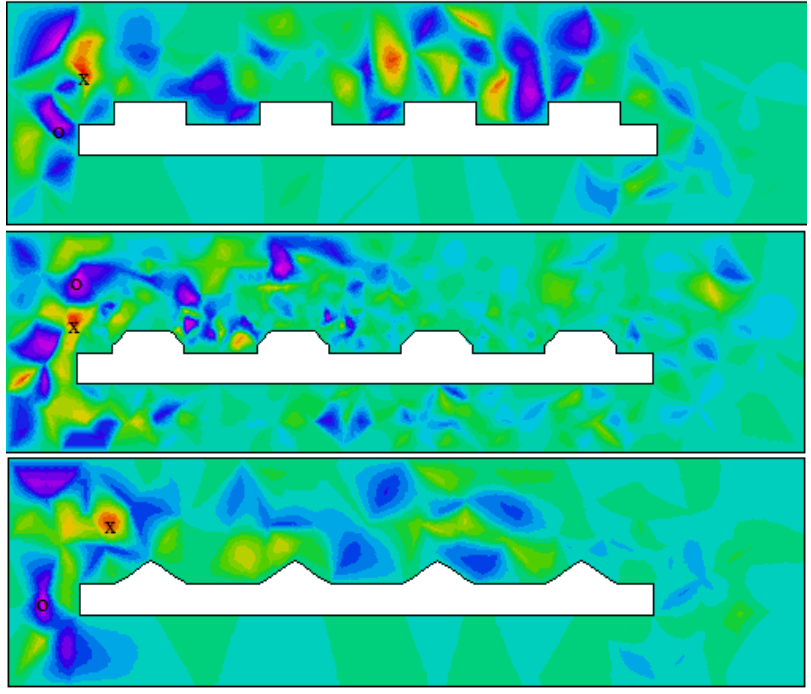

b) Stretching sheet with four fins

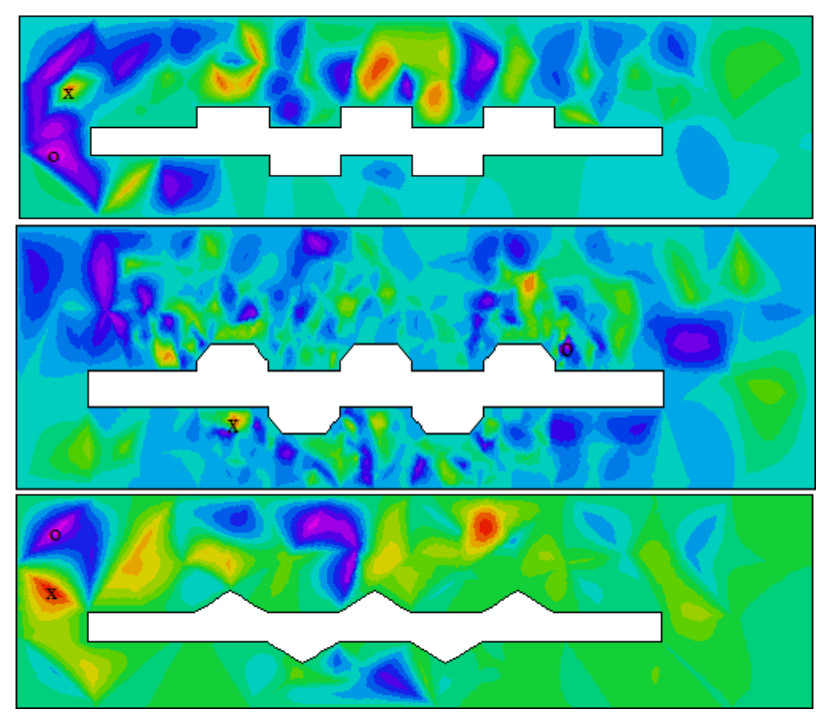

c) Stretching sheet with five fins

Fig. 6: comparison of different values of concentration for rectangular and triangular and chamfer fins

The above images indicate the thickness of the fluid around the thickness of the plane. in the first column from left to right, we compare the different concentrations of fluid flow over the fins. As it is observed, the fluid concentration around the chamfer fins has less value than other modes. The concentration of concentration around the fins is in a state of equilibrium and has a moderate value. in the first row from left to right, the fluid flow around the upper fins has lower values and around the lower fins of the plate, the minimum value is minimum. In front of the screen, the fluid concentration is lower than the end of the page. among the figures derived from different scenarios, the shape of triangular blade on the surface has the highest amount of fluid concentration in the plate entrance area. In the beginning, the concentration is about 5, and gradually the concentration is reduced.

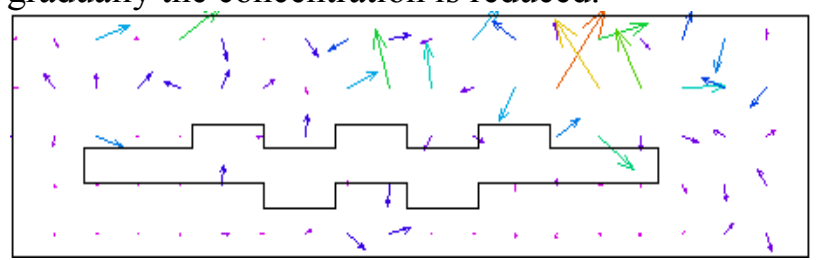

a) Stretching sheet with rectangular fins

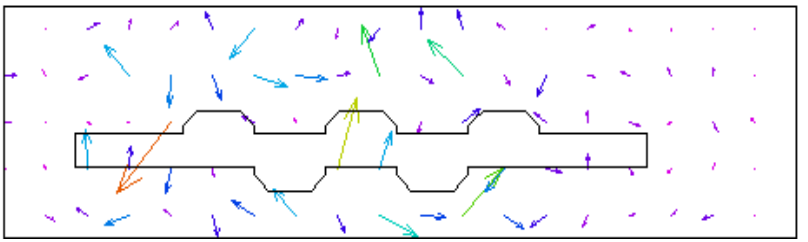

b) Stretching sheet with chamfer fins 


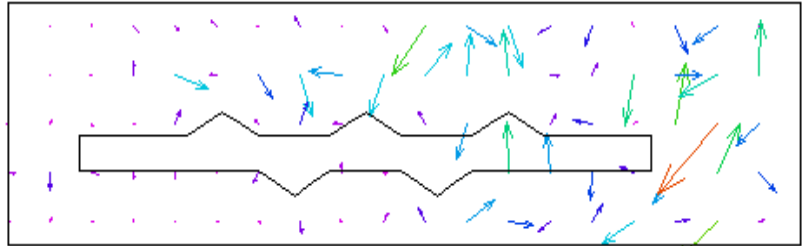

c) Stretching sheet with triangular fins

Fig. 7: comparison of different values of vector grid of Temperature for rectangular and triangular and chamfer fins.

The above cases show the contours of the fluid motion vectors around the plane surface and the fins. In the vicinity of rectangular fins, the fluid flow particles have a lower temperature and the size of the small temperature vector is small and the higher the level of the fins, the bigger the size of the temperature vector. in triangular fins, the fluid temperature vector around the fins has more intensity than other modes and the temperature gradient around it is larger than the previous one and the fluid flow at the end of the plate also has more temperature than the Rectangular fins. in the third form, from the first row containing chamfer fins, the density of the temperature vectors at the bottom of the plane and around the lower fins of the page is much higher than on the screen.
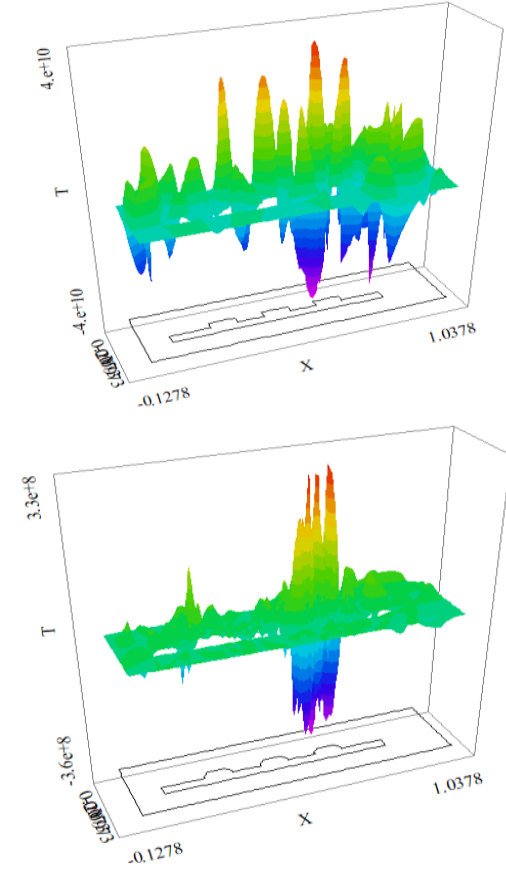

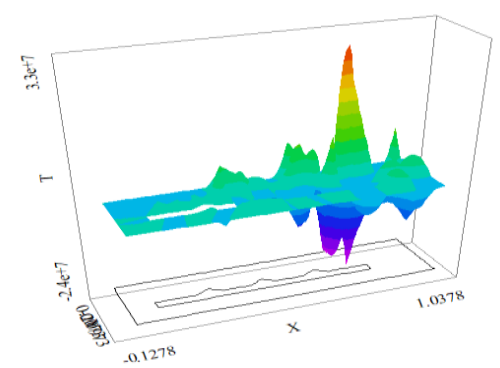

a) Stretching sheet with three fins
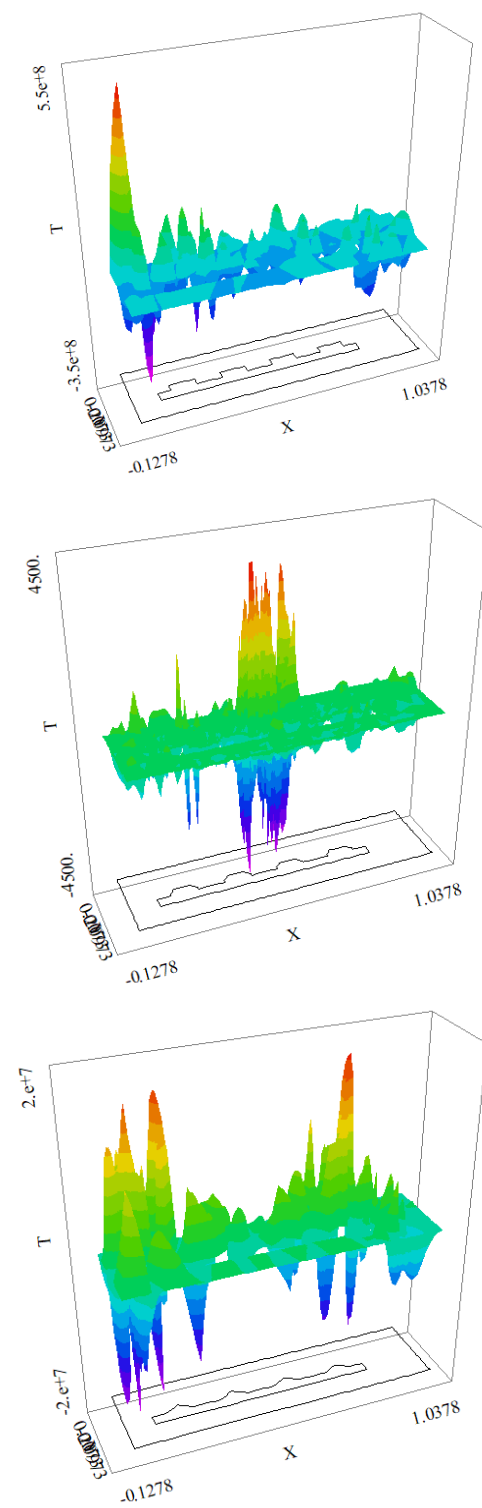

b) Stretching sheet with four fins 

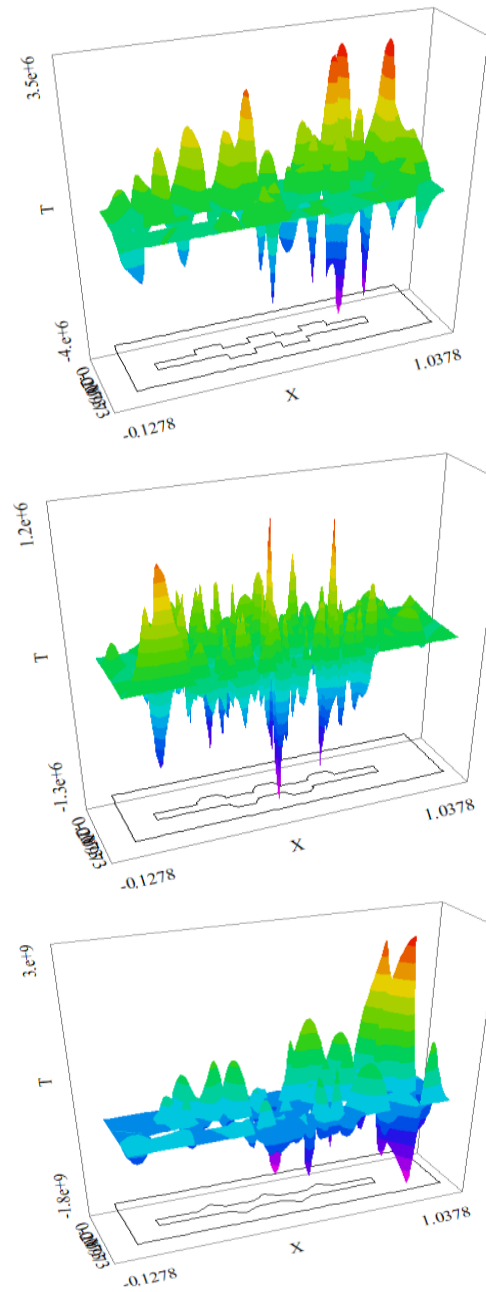

c) Stretching sheet with five fins

Fig. 8: surface comparison of different values of Temperature for rectangular and triangular and chamfer fins

The above shapes indicate the variation of fluid temperature across the plates. First, we describe the first line. fluid flow is balanced in most parts surrounding the triangular fins and the maximum temperature of value 3.5 occurs in the third fin of the plate. The lowest temperature is observed below the screen. Now, we look at the comments of the second row of the table. In the case of fluid flow passing over the triangular Fins, the temperature of the fluid flow is very low and in the range of numbers- 1 to- 2 . by passing the fluid through the first and second fins, the temperature increases and the peak in the third blade reaches a maximum. The third row of the above table is related to the chamfer fins. in this type of fins, the fluid flow temperature in the first and second fin has the highest value. and it gets less heat in the third blade and balanced and The value of it is 1 .

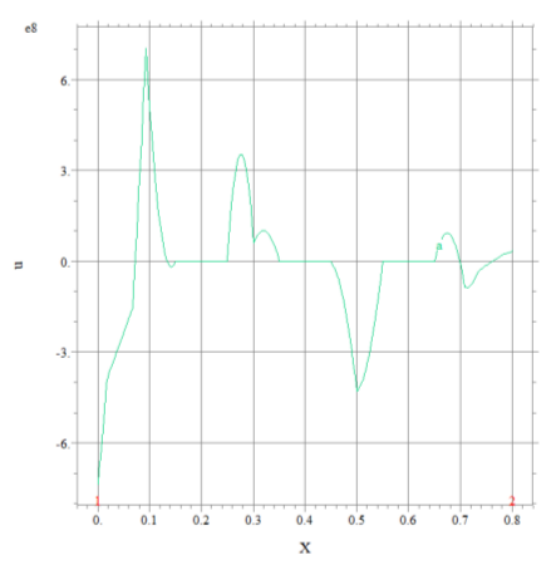

a) Stretching sheet with rectangular fins

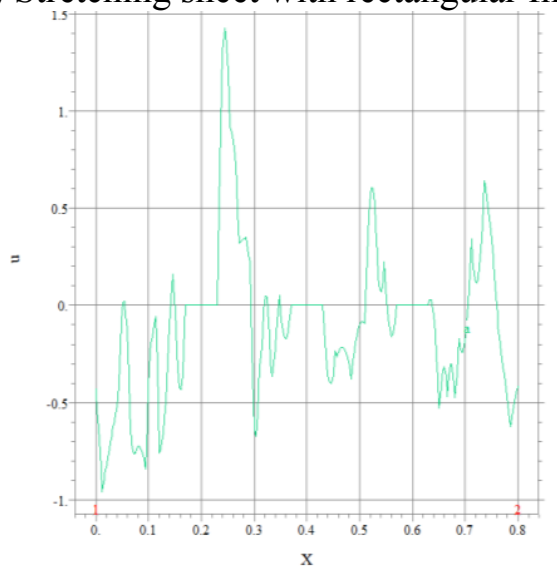

b) Stretching sheet with chamfer fins

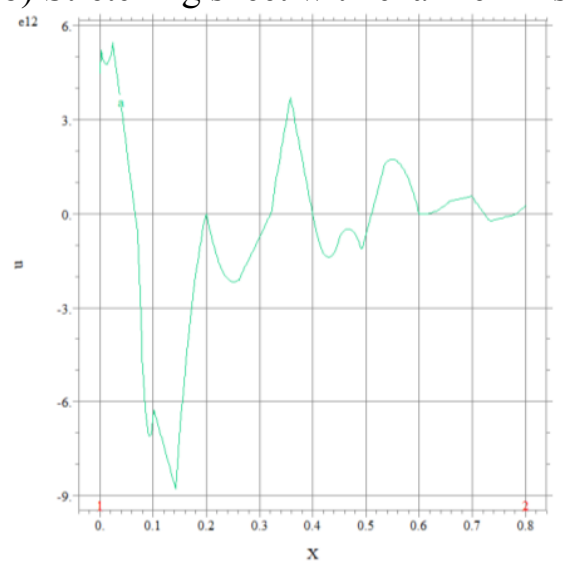

c) Stretching sheet with triangular fins

Fig. 9: comparison of different values of velocity (u) for rectangular and triangular and chamfer fins.

The above figures display the changes in fluid flow velocity changes at the 3 fins levels at the top of the screen. In the left figure, the velocity of the fluid flow in the direction $\mathrm{x}$ around the first fin is large and It reaches the value $u=7$. Then, the flow rate is reduced, and the peak in the third fin reaches the lowest in the number 0.5 . for the chamfer fins, the rate of velocity changes is much higher than the other two modes. The velocity fluctuation around the fins is very high in the chamfer mode, where the 
maximum value is $\mathrm{u}=1.7$ and its lowest values in $\mathrm{u}=-0.9$. The maximum velocity is around the first fin in the range $\mathrm{x}=0.2$ to $\mathrm{x}=0.3$ and is the lowest in the second and third fin. for triangular fins, the rate of fluid velocity at first decreases from the upper bound in the first fin and reaches the value $\mathrm{u}=-7$ but by crossing the level of the second fin reaches the value $u=4$. in comparison to the rate of fluid velocity in $\mathrm{x}$ for surfaces with different fins, we concluded that the maximum rate drop rate at the end of the plane is chamfer. In rectangular mode, the maximum velocity rate is observed around the first fin and the lowest rate of velocity around the first fin is also related to chamfer mode.

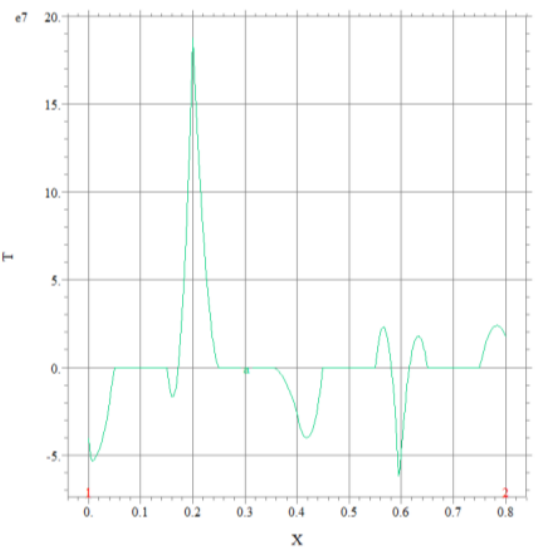

a) Stretching sheet with rectangular fins

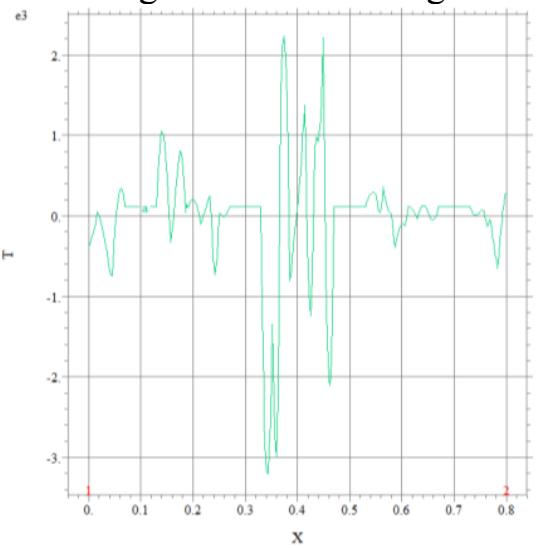

b) Stretching sheet with chamfer fins

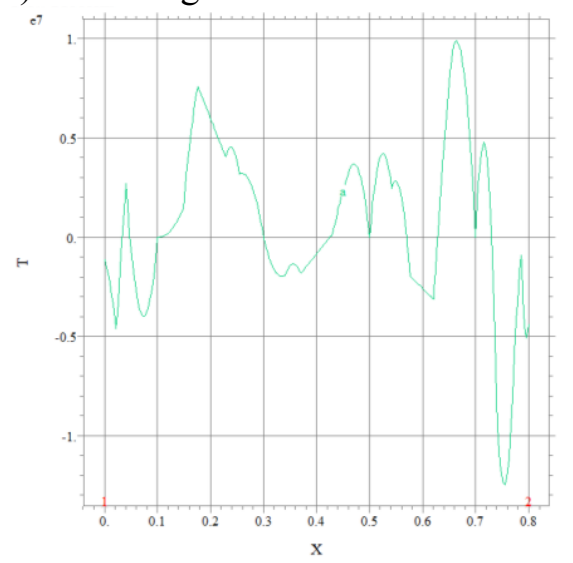

c) Stretching sheet with triangular fins

Fig. 10: comparison of different values of Temperature for rectangular and triangular and chamfer fins.

In the first figure, the maximum heat and temperature is observed around the first fin, which is $\mathrm{T}=19$. With fluid flow over the top of the fins, the temperature drop is very high and in $\mathrm{x}=0.8$, it will reach $\mathrm{T}=0.2$ in the fourth blade. In triangular fins, maximum temperature is observed around the fourth fin. In this case the maximum temperature $(T=1)$ occurs at $\mathrm{x}=0.6$ and The lowest temperature is at the end of the plane at point $\mathrm{x}=0.8$. in the case of chamfer fins, the maximum temperature variation is observed. In this fins, the maximum temperature of $\mathrm{T}=2.5$ and minimum temperature is $\mathrm{T}=3$. in general, we conclude that the temperature flow around the rectangular fins has a maximum value than 2 other modes.

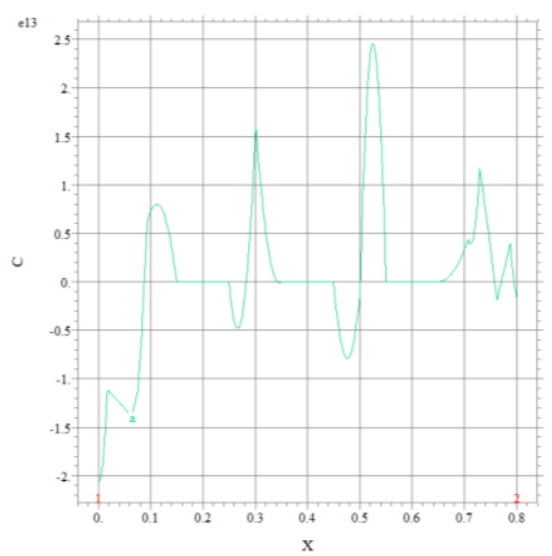

a) Stretching sheet with rectangular fins

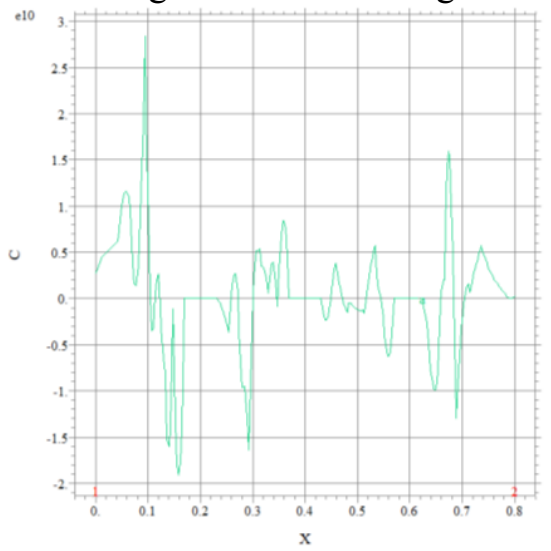

b) Stretching sheet with chamfer fins 


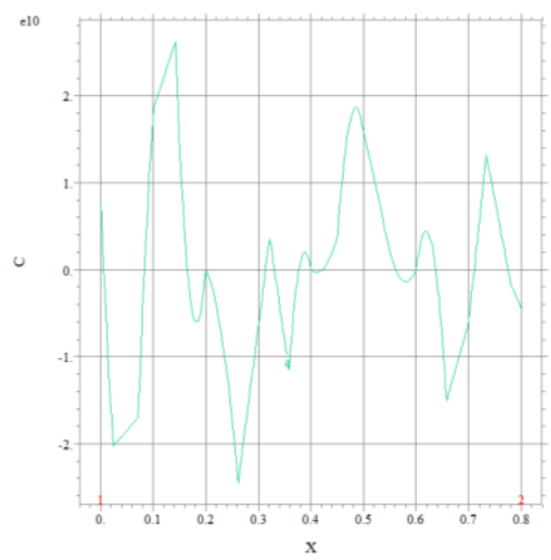

c) Stretching sheet with triangular fins

Fig. 11: comparison of different values of concentration for rectangular and triangular and chamfer fins.

High figures show the amount of fluid flow concentration around the hot plate. The maximum amount of fluid concentration has been observed around the first fin of chamfer mode in range of $\mathrm{x}=0.05$ to $\mathrm{x}=0.1$. In general, the fluid concentration around the triangular fins is higher than other modes. The maximum amount of fluid concentration is found in the triangular fins on the surface. Their concentration from the first fin reaches a value of 2.5 and in the last fin at a value of 1.4 .

\section{Conclusion}

In this paper, we investigated the flow of fluid flowing through the fins plate under the influence of the magnet. The fins were on the board and the end of the plate. Finite element Model is applied for investigation of fluid flow over a stretching sheet in existence of magnetic field. Finite element method is applied to find the influence of melting heat transfer on fluid flow behavior over a stretching sheet in presence of magnetic field.

- In the case of chamfer fins, the maximum temperature variation is observed. In this fins, the maximum temperature of $\mathrm{T}=2.5$ and minimum temperature is $T=3$. in general, we conclude that the temperature flow around the rectangular fins has a maximum value than 2 other modes.

- In the case of fluid flow passing over the triangular Fins, the temperature of the fluid flow is very low and in the range of numbers -1 to- 2 . by passing the fluid through the first and second fins, the temperature increases and the peak in the third blade reaches a maximum.
- In triangular fins, the fluid temperature vector around the fins has more intensity than other modes and the temperature gradient around it is larger than the previous one and the fluid flow at the end of the plate also has more temperature than the Rectangular fins.

- High figures show the amount of fluid flow concentration around the hot plate. The maximum amount of fluid concentration has been observed around the first fin of chamfer mode in range of $x=0.05$ to $x=0.1$.

- In general, the fluid concentration around the triangular fins is higher than other modes. the maximum amount of fluid concentration is found in the triangular fins on the surface. Their concentration from the first fin reaches a value of 2.5 and in the last fin at a value of 1.4.

\section{References:}

[1] Peiravi, M. M., Alinejad, J., Domairry Ganji, D., and Maddah, S., "Numerical Study of Fins Arrangement and Nanofluids Effects On ThreeDimensional Natural Convection in The Cubical Enclosure," Transp Phenom Nano Micro Scales, Vol. 7, No. 2, pp. 97-112, 2019.

[2] Peiravi, M. M., Alinejad, J., Domairry Ganji, D., and Maddah, S., "3D Optimization of Baffle Arrangement in A Multi-Phase Nanofluid Natural Convection Based On Numerical Simulation," International Journal of Numerical Methods for Heat and Fluid Flow, Vol. 30, No. 5, 2583-2605. 2019.

[3] Adamo, Cristina Battesini, "Fast and straightforward in-situ synthesis of gold nanoparticles on a thread-based microfluidic device for application in surface-enhanced Raman scattering detection." Micro chemical Journal Vol. 156, 104985, 2020.

[4] Yen, Ching-Yu, "Accuracy of cone beam computed tomography in measuring thicknesses of hard-tissue-mimicking material adjacent to different implant thread surfaces" Journal of dental sciences, Vol. 14, 119-125, 2019.

[5] Domairry Ganji, D., Peiravi, M. M., and Abbasi, M., "Evaluation of The Heat Transfer Rate Increases in Retention Pools Nuclear Waste. International Journal of Nano Dimension," International Journal of Nano Dimension, Vol. 6, No. 4, pp. 385-398, 2015.

[6] Seyyedi, Seyyed Masoud, "Investigation of entropy generation in a square inclined cavity using control volume finite element method with aided quadratic Lagrange interpolation 
functions." International Communications in Heat and Mass Transfer 110, 104398, 2020.

[7] Peiravi, M. M., and Alinejad, J., "Nano Particles Distribution Characteristics in MultiPhase Heat Transfer Between 3D Cubical Enclosures Mounted Obstacles," Alexandria Engineering Journal, Vol. 60. No. 6, pp. 50255038, 2021.

[8] Neuman, Shlomo P., and Paul A. Witherspoon. "Finite element method of analyzing steady seepage with a free surface." Water Resources Research Vol. 6, 3, 889-897, 1970.

[9] Sindhu, S., B. J. Gireesha, and D. D. Ganji. "Simulation of $\mathrm{Cu}: \gamma-\mathrm{ALOOH} /$ Water in a microchannel heat sink by dint of porous media approach." Case Studies in Thermal Engineering Vol. 21, 100723, 2020.

[10] Peiravi, M. M., and Alinejad, J.,"Numerical Analysis of Secondary Droplets Characteristics Due to Drop Impacting On 3D Cylinders Considering Dynamic Contact Angle," Meccanica,Vol. 55. No. 10. 1975-2002.2020.

[11] Peiravi, M. M., and Alinejad, J., "Hybrid Conduction, Convection and Radiation Heat Transfer Simulation in A Channel with Rectangular Cylinder," Journal of Thermal Analysis and Calorimetry, Vol. 140. No. 6. 2733-2747. 2019.

[12] Ghadikolaei, S. S., "MHD boundary layer analysis for micropolar dusty fluid containing Hybrid nanoparticles $(\mathrm{Cu}-\mathrm{Al} 2 \mathrm{O} 3)$ over a porous medium." Journal of Molecular Liquids 268 (2018): 813-823.

\section{Creative Commons Attribution License 4.0 (Attribution 4.0 International, CC BY 4.0)}

This article is published under the terms of the Creative Commons Attribution License 4.0

https://creativecommons.org/licenses/by/4.0/deed.en_US 